\title{
miR-20a is an independent prognostic factor in colorectal cancer and is involved in cell metastasis
}

\author{
GUANG-JUN ZHANG ${ }^{*}$, YU LI ${ }^{*}$, HE ZHOU, HUA-XU XIAO and TONG ZHOU \\ The First Department of General Surgery, Institute of Hepato-Biliary-Pancreas and Intestinal Disease, \\ The Affiliated Hospital of North Sichuan Medical College, Nanchong, Sichuan 637000, P.R. China
}

Received July 9, 2013; Accepted March 17, 2014

DOI: $10.3892 / \mathrm{mmr} .2014 .2144$

\begin{abstract}
Accumulating evidence indicates that dysregulated microRNAs (miRNAs) are involved in cancer development, progression and metastasis. miR-20a was found to be involved in invasion and epithelial-mesenchymal transition (EMT) programs, with its aberrant expression having been observed in a variety of malignant tumors. However, the molecular mechanisms underlying the role of miR-20a in colorectal cancer (CRC) development remain to be fully elucidated. In the present study, the expression of miR-20a was compared between $\mathrm{CRC}$ tissue samples and the normal adjacent mucosa using quantitative polymerase chain reaction. The association of miR-20a expression with clinicopathological characteristics was assessed using appropriate statistical analysis. The migration and invasion of SW480 cells was examined following transfection of the cells with either miR-20a precursor or a negative control miRNA precursor. The effect of miR-20a on the EMT in CRC cells in vitro was also analyzed. The regulatory effect of miR-20a on SMAD family member 4 (SMAD4) was evaluated using a dual-luciferase reporter assay. Relative expression levels of miR-20a were significantly higher in CRC tissue than those in the normal adjacent mucosa, and high expression of miR-20a correlated with lymph node metastases and distant metastases. Kaplan-Meier analysis indicated that patients with increased miR-20a levels exhibited unfavorable overall survival. Furthermore, multivariate analysis showed that miR-20a was an independent prognostic factor. The transfection of SW480 CRC cells with miR-20a promoted migration and invasion in vitro, and the upregulation of miR-20a induced
\end{abstract}

Correspondence to: Professor Tong Zhou, The First Department of General Surgery, Institute of Hepato-Biliary-Pancreas and Intestinal Disease, The Affiliated Hospital of North Sichuan Medical College, 63 Wenhua Road, Nanchong, Sichuan 637000, P.R. China

E-mail: zhoutong0088@163.com

*Contributed equally

Key words: miR-20a, colorectal cancer, epithelial-mesenchymal transition, metastasis, prognostic factor
EMT in CRC cells. An inverse correlation between the levels of miR-20a and SMAD4 was observed in patients with CRC. Overexpression of miR-20a in CRC cells decreased SMAD4 expression and decreased SMAD4-driven luciferase reporter activity. The present study revealed that miR-20a was an independent prognostic factor in CRC. Furthermore, miR-20a induced EMT and regulated migration and invasion of SW480 cells, at least in part via suppression of SMAD4 expression. The present study suggests that miR-20a may serve as a novel prognostic marker and therapeutic target for CRC.

\section{Introduction}

Colorectal cancer (CRC) is the third most common malignancy and the fourth most frequent cause of cancer-related mortality worldwide (1). Despite numerous advances in treatment, patients with CRC still have an unfavorable prognosis, and $25-40 \%$ of patients develop recurrence following surgery (2). Therefore, it is crucial to enhance the understanding of the mechanisms involved in CRC metastasis and to identify potential prognostic biomarkers and therapeutic targets.

Epithelial-mesenchymal transition (EMT) is a key step towards cancer metastasis. Loss of E-cadherin expression is a hallmark of the EMT process and is likely to be required for enhanced tumor cell motility (3-5). Epithelial cells lose their epithelial characteristics and acquire mesenchymal characteristics by the downregulation of E-cadherin (6). EMT has a crucial role in tumor cell invasion and migration in numerous types of cancer, including CRC (7). Inducers of EMT include certain protein polypeptides, transcription factors, growth factors and microRNAs (miRNAs) (8).

miRNAs are small, noncoding RNA gene products of $\sim 22$ nucleotides that are found in a variety of organisms. miRNAs have key roles in regulating the translation and degradation of mRNAs through base pairing to partially complementary sites, predominantly in the 3'-untranslated regions (UTRs) of mRNAs (9-11). It is well known that miRNAs have important regulatory functions in basic biological processes, including development, cellular differentiation, proliferation and apoptosis, which affect major biological systems, including stemness, immunity and cancer $(12,13)$. Data suggest that dysregulation of miRNAs is an important step in the pathogenesis, from initiation to metastasis, of numerous types of cancer, including CRC (14-16). 
Accumulating evidence has demonstrated that the induction of EMT and aberrant expression of miRNAs are associated with tumorigenesis, tumor progression, metastasis and relapse in certain cancers, including CRC $(17,18)$.

Recently, miR-20a has been shown to be deregulated in several types of cancer (19-21). In particular, miR-20a has a key role in the regulation of tumor proliferation, metastasis and EMT (19-24). Chai et al (25) reported that the overexpression of miR-20a contributed to the resistance of colorectal adenocarcinoma to chemotherapeutics. However, the clinical and biological roles of miR-20a in CRC remain to be fully elucidated.

The aim of the present study was to assess the clinical significance of miR-20a in CRC and to investigate the effects of miR-20a on the migration, invasion and EMT of CRC cells, and to further discuss the mechanisms of action of miR-20a by identifying its potential target gene.

\section{Materials and methods}

Patients and tissue samples. Paired tissue specimens (tumor and adjacent normal mucosa) were obtained from 86 patients with primary CRC who underwent surgery without preoperative treatment at the First Department of General Surgery, the Affiliated Hospital of North Sichuan Medical College (Nanchong, China), from 2005 to 2008, following receipt of their informed consent. All tissue samples were immediately frozen in liquid nitrogen and stored at $-80^{\circ} \mathrm{C}$ for subsequent analysis. Specimens were stained with hematoxylin and eosin and examined histopathologically. Sections that consisted of $>80 \%$ carcinoma cells were used to prepare the total RNA. Clinicopathological information, including age, gender, tumor size, histological type, depth of invasion, location, lymph node metastasis, lymphatic invasion and distant metastasis, was available for all patients. The study was approved by the Medical Ethics Committee of North Sichuan Medical College.

Cell culture. The human SW480 CRC cell line was obtained from the American Type Culture Collection (Manassas, VA, USA) and cultured in Dulbecco's Modified Eagle's medium supplemented with $10 \%$ fetal bovine serum, $100 \mathrm{U} / \mathrm{ml}$ penicillin and $100 \mathrm{mg} / \mathrm{ml}$ streptomycin at $37^{\circ} \mathrm{C}$ in a humidified atmosphere of $5 \% \mathrm{CO}_{2}$.

Quantitative polymerase chain reaction ( $q P C R)$. Total RNA was extracted using TRIzol ${ }^{\circledR}$ reagent (Invitrogen Life Technologies, Carlsbad, CA, USA) for both miR-20a and SMAD family member 4 (SMAD4) mRNA analyses. For detection of miR-20a expression, qPCR was performed using the QuantiMir RT kit (System Biosciences, Mountain View, CA, USA) according to the manufacturer's instructions with miR-20a-specific primers (Applied Biosystems, Foster City, CA, USA). The amplification profile was denaturation at $95^{\circ} \mathrm{C}$ for $10 \mathrm{~min}$ followed by 45 cycles of denaturation at $95^{\circ} \mathrm{C}$ for $15 \mathrm{sec}$, annealing at $60^{\circ} \mathrm{C}$ for $30 \mathrm{sec}$ and extension at $72^{\circ} \mathrm{C}$ for $1 \mathrm{~min}$. For the detection of SMAD4 mRNA expression, qPCR was performed using SYBR ${ }^{\circledR}$ Green PCR Master Mix (Applied Biosystems). The primers for SMAD4 were as follows: 5'-TGGCCCAGGATCAGTAGGT-3' and 5'-CATCAACACCAATTCCAGCA-3'. The primers for $\beta$-actin were: 5'-CCAAGGCCAACCGCGAGAAGATGAC-3' and 5'-AGGGTACATGGTGGTGCCGCCAGAC-3'. The amplification profile was denaturation at $95^{\circ} \mathrm{C}$ for $30 \mathrm{~min}$, followed by 40 cycles of denaturation at $95^{\circ} \mathrm{C}$ for $10 \mathrm{sec}$, annealing at $60^{\circ} \mathrm{C}$ for $10 \mathrm{sec}$ and extension at $72^{\circ} \mathrm{C}$ for $1 \mathrm{~min}$. U6 and $\beta$-actin were used as internal controls. Relative gene expression was calculated using the equation $2^{-\Delta \mathrm{CT}}$. The fold-change of gene expression was calculated using the equation $2^{-\Delta \Delta \mathrm{CT}}$. All reactions were run in triplicate.

Transfection of miRNA. Ectopic expression of miR-20a in cells was achieved by transfection with Pre-miR ${ }^{\mathrm{TM}}$ miR-20a precursor (pre-miR-20a; Applied Biosystems) using Lipofectamine 2000 (Invitrogen Life Technologies). A total of $2 \times 10^{5}$ cells were seeded into each well of a six-well plate and transfected for 24 or $48 \mathrm{~h}$. Transfected cells were used in further assays or RNA/protein extraction.

Migration and invasion assays. To assess cell migration and invasion, $5 \times 10^{4}$ SW480 cells transfected with either pre-miR-20a or the negative control miRNA precursor (pre-miR-nc; Applied Biosystems) were seeded into Transwell chambers (8.0- $\mu \mathrm{m}$ pore size; Corning, Inc., New York, NY, USA) uncoated or coated with Matrigel ${ }^{\mathrm{TM}}$ (BD Biosciences, Bedford, MA, USA). Medium containing 10\% fetal bovine serum in the lower chamber served as the chemoattractant. Following incubation for $48 \mathrm{~h}$ at $37^{\circ} \mathrm{C}$ in a humidified incubator with $5 \% \mathrm{CO}_{2}$, cells that did not migrate through the pores were mechanically removed using a cotton swab. The migrated cells attached to the bottom of the membrane insert were fixed with methanol at room temperature for $5 \mathrm{~min}$ and stained with hematoxylin. The number of migrated or invaded cells on the lower surface of the membrane was then counted under a microscope at a magnification of $x 400$.

Western blot analysis. To isolate the proteins, cells collected from the six-well plates were washed twice with phosphate-buffered saline and lysed in radioimmunoprecipitation assay lysis buffer (ProMab Biotechnologies, Inc., Richmond, CA, USA). Lysates were kept on ice for $30 \mathrm{~min}$ and then centrifuged at $13,000 \mathrm{x}$ g for $30 \mathrm{~min}$. The supernatant was collected and then $20 \mu \mathrm{g}$ of each of the proteins was separated by $10 \%$ SDS-PAGE and transferred to nitrocellulose membranes. Following blocking in 5\% skimmed milk, the membranes were incubated with the respective antibodies: Mouse anti-E-cadherin (1:1,000; BD Biosciences, San Jose, CA, USA), mouse anti-vimentin (1:500; Santa Cruz Biotechnology, Inc., Santa Cruz, CA, USA), mouse anti-SMAD4 (1:500; Santa Cruz Biotechnology, Inc.) and mouse anti-GAPDH (1:1,000; Santa Cruz Biotechnology, Inc.) overnight at $4^{\circ} \mathrm{C}$. Following incubation with the appropriate secondary antibody, the bands were visualized using ECL-Plus ${ }^{\mathrm{TM}}$ reagents (GE Healthcare Bio-Science Corp., Piscataway, NJ, USA). The density of the SMAD4 and GAPDH bands was measured using Image $\mathbf{J}$ software (National Institutes of Health, Bethesda, MD, USA), and values were normalized to the densitometric values of GAPDH in each sample. Fold-changes in the amount of protein were then calculated for the experimental sets compared with the control. 


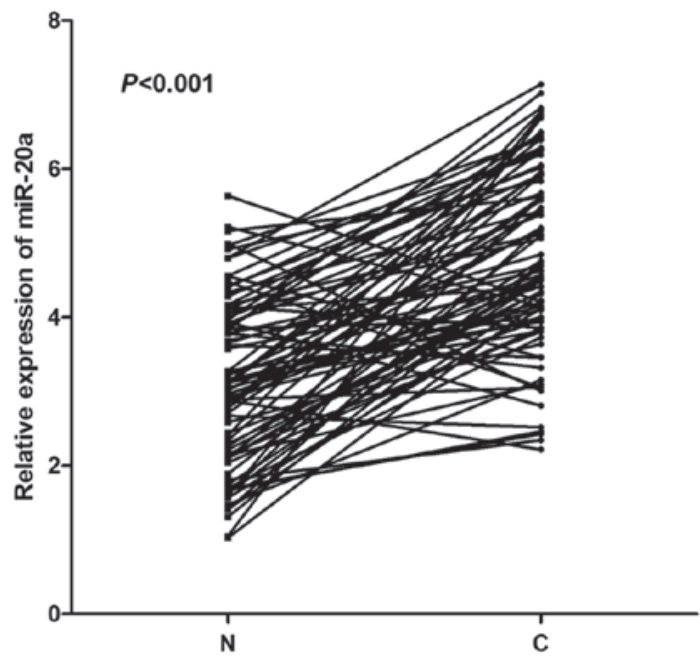

Figure 1. Comparison of miR-20a expression levels between colorectal cancer tissue (C) and adjacent normal mucosa (N). miR-20a expression levels were normalized using U6 as an internal control. miR-20a expression levels were higher in cancer tissue than in normal adjacent mucosa $(\mathrm{P}<0.001)$. miR-20a, microRNA-20a.

Luciferase assay. For the luciferase reporter experiments, the wild-type and mutated 3'UTRs of SMAD4 mRNA were subcloned into the XhoI and NotI sites of the psicheck-2 vector (Promega Corp., Madison, WI, USA) and the new wild-type and mutant vectors were referred to as psicheck-2-SMAD4-WT and psicheck-2-SMAD4-MUT, respectively. The following primers were used to amplify specific fragments: WT sense, 5'-CACAACTCGAGCACTGTTCTGCAAAGGTGGC-3' and WT antisense, 5'-AAGGAAAAAAGCGGCCGCGACCTT CTGAGCAAGGCAGT-3'; MUT sense, 5'-CTTTTCGTG AAATGAGTCCAATCTCAGTGATGAGG-3' and MUT antisense, 5'-CTCATTTCACGAAAAGAAAAAAAAAAT CTTAAAAATCA-3'. For the reporter assay, HEK 293 T cells (ATCC, Manassas, VA, USA) were seeded onto 24-well plates at $2 \times 10^{4}$ cells/well and transfected with $200 \mathrm{ng}$ psicheck-2-SMAD4-WT or -SMAD4-MUT and $40 \mathrm{nM}$ pre-miR-20a or -nc using Lipofectamine 2000 (Invitrogen Life Technologies). Firefly luciferase was used to normalize the Renilla luciferase. Following transfection for $48 \mathrm{~h}$, cells were harvested and assayed with the Dual-Luciferase Reporter Assay System (Promega Corp.) according to the manufacturer's instructions.

Bioinformatics analysis. The miRNA targets predicted by computer-aided algorithms were obtained from TargetScan (http://www.targetscan.org) and miRanda (http://www.microrna.org).

Statistical analysis. Values are expressed as the mean \pm standard deviation from at least three separate experiments performed in triplicate. The gene expression levels in CRC tissue samples were compared with those in normal adjacent mucosa using the Wilcoxon signed-rank test. Measurement data were analyzed using the two-tailed Student's t-test, while categorical data were studied using the $\chi^{2}$ test. The correlation between miR-20a levels and SMAD4 expression was analyzed using Pearson's correlation. The postoperative survival rate
Table I. Association of miR-20a expression with clinicopathological factors of patients with colorectal cancer.

\begin{tabular}{|c|c|c|c|}
\hline \multirow[b]{2}{*}{ Variable } & \multicolumn{2}{|c|}{ miR-20a expression } & \multirow[b]{2}{*}{ P-value } \\
\hline & Low, $n=43$ & High, $n=43$ & \\
\hline Age (years) & $62.2 \pm 13.4$ & $63.3 \pm 14.1$ & 0.702 \\
\hline Gender & & & 0.506 \\
\hline Male (n) & 25 & 28 & \\
\hline Female (n) & 18 & 15 & \\
\hline Tumor size & & & 0.176 \\
\hline$\leq 5 \mathrm{~cm}(\mathrm{n})$ & 37 & 32 & \\
\hline$>5 \mathrm{~cm}(\mathrm{n})$ & 6 & 11 & \\
\hline Histological type & & & 0.181 \\
\hline Well, moderate (n) & 30 & 24 & \\
\hline Poor, mucinous (n) & 13 & 19 & \\
\hline Depth of invasion & & & 0.078 \\
\hline $\mathrm{T} 1, \mathrm{~T} 2(\mathrm{n})$ & 21 & 13 & \\
\hline $\mathrm{T} 3, \mathrm{~T} 4$ (n) & 22 & 30 & \\
\hline Location & & & 0.651 \\
\hline Colon (n) & 16 & 14 & \\
\hline Rectum (n) & 27 & 29 & \\
\hline Lymph node metastasis & & & $0.017^{\mathrm{a}}$ \\
\hline Absent (n) & 25 & 14 & \\
\hline Present (n) & 18 & 29 & \\
\hline Lymph node invasion & & & 0.195 \\
\hline Absent (n) & 26 & 20 & \\
\hline Present (n) & 17 & 23 & \\
\hline Distant metastasis & & & $0.035^{\mathrm{a}}$ \\
\hline Absent (n) & 40 & 33 & \\
\hline Present (n) & 3 & 10 & \\
\hline
\end{tabular}

${ }^{\mathrm{a}} \mathrm{P}<0.05$. n, number of patients; miR-20a, microRNA-20a; well, well-differentiated; moderate, moderately differentiated; poor, poorly differentiated.

was analyzed using the Kaplan-Meier method, and differences in survival rates were assessed using the log-rank test. A Cox proportional hazards model was used for multivariate analysis. The findings were considered significant at a P-value of $<0.05$. All statistical analyses were performed using the SPSS 16.0 software (SPSS, Inc., Chicago, IL, USA).

\section{Results}

Expression of miR-20a in CRC tissue samples and normal adjacent mucosa. The expression levels of miR-20a in tumor and normal tissue samples from 86 patients are shown in Fig. 1. Most tumor tissue samples from patients with CRC $(75 / 86 ; 87.21 \%)$ showed elevated levels of miR-20a, unlike the corresponding normal tissue samples $(\mathrm{P}<0.001)$.

Correlation between miR-20a expression and clinicopathological characteristics in CRC. The expression levels 
Table II. Univariate analysis of clinicopathological factors for overall survival.

\begin{tabular}{|c|c|c|c|c|}
\hline Variable & $\mathrm{n}$ & Hazard ratio & $95 \% \mathrm{CI}$ & P-value \\
\hline Age (years) & & & $0.715-3.173$ & 0.282 \\
\hline$\leq 60$ & 36 & 1 & & \\
\hline$>60$ & 50 & 1.506 & & \\
\hline Gender & & & $0.380-1.700$ & 0.568 \\
\hline Male & 53 & 1 & & \\
\hline Female & 33 & 0.804 & & \\
\hline Tumor size & & & $1.078-5.646$ & $0.032^{\mathrm{a}}$ \\
\hline$\leq 5 \mathrm{~cm}$ & 69 & 1 & & \\
\hline$>5 \mathrm{~cm}$ & 17 & 2.468 & & \\
\hline Histological & & & $1.573-7.160$ & $0.002^{\mathrm{b}}$ \\
\hline Well, moderate & 54 & 1 & & \\
\hline Poor, mucinous & 32 & 3.356 & & \\
\hline Depth of invasion & & & $1.064-5.913$ & $0.035^{\mathrm{a}}$ \\
\hline $\mathrm{T} 1, \mathrm{~T} 2$ & 34 & 1 & & \\
\hline $\mathrm{T} 3, \mathrm{~T} 4$ & 52 & 2.187 & & \\
\hline Location & & & $0.796-3.570$ & 0.173 \\
\hline Colon & 30 & 1 & & \\
\hline Rectum & 56 & 1.686 & & \\
\hline Lymph node metastasis & & & $2.447-20.459$ & $<0.001^{\mathrm{b}}$ \\
\hline Absent & 39 & 1 & & \\
\hline Present & 47 & 7.076 & & \\
\hline Lymph node invasion & & & $1.072-4.931$ & $0.032^{\mathrm{a}}$ \\
\hline Absent & 46 & 1 & & \\
\hline Present & 40 & 2.299 & & \\
\hline Distant metastasis & & & $4.609-26.247$ & $<0.001^{\mathrm{b}}$ \\
\hline Absent & 73 & 1 & & \\
\hline Present & 13 & 10.998 & & \\
\hline miR-20a & & & $1.112-5.253$ & $0.026^{\mathrm{a}}$ \\
\hline Low & 43 & 1 & & \\
\hline High & 43 & 2.417 & & \\
\hline
\end{tabular}

${ }^{\mathrm{a}} \mathrm{P}<0.05,{ }^{\mathrm{b}} \mathrm{P}<0.01 ; \mathrm{n}$, number of patients; CI, confidence interval; well, well-differentiated; moderate, moderately differentiated; poor, poorly differentiated; miR-20a, microRNA-20a.

of miR-20a were categorized as low or high relative to the median value (4.65). High expression rates of miR-20a in CRC tissue samples with respect to several standard clinicopathological characteristics are listed in Table I. High expression of miR-20a was significantly correlated with lymph node and distant metastases $(\mathrm{P}<0.05$, Table I). However, no significant correlation was observed between miR-20a expression and other clinicopathological features, including age, gender, tumor size, histological type, depth of invasion, tumor location and lymph node invasion $(\mathrm{P}>0.05$, Table I).

Correlation between miR-20a expression and prognosis of patients with CRC. Overall survival curves were plotted according to miR-20a mRNA expression levels using the Kaplan-Meier method. As shown in Fig. 2, the overall survival rate was significantly lower in patients with high miR-20a expression than that in patients with low expression levels $(\mathrm{P}=0.021)$. Univariate analysis using the Cox proportional hazards model identified seven prognostic factors: Histological type, tumor size, depth of invasion, lymph node metastasis, lymph node invasion, distant metastasis and miR-20a expression. The other clinicopathological features (age, gender and location) were not statistically significant prognostic factors (Table II). A multivariate analysis of the prognostic factors using the Cox proportional hazards model confirmed that high miR-20a expression was a significant independent predictor of low survival rates of patients with $\mathrm{CRC}(\mathrm{P}=0.045)$ in addition to the presence of lymph node metastasis $(\mathrm{P}=0.025)$ and distant metastasis $(\mathrm{P}<0.001)$ (Table III).

miR-20a regulates $C R C$ cell invasion and migration in vitro. To investigate the role of miR-20a in CRC metastasis, the 
Table III. Multivariate analysis of clinicopathological factors for overall survival.

\begin{tabular}{lccc}
\hline Variable & Hazard ratio & $95 \%$ CI & P-value \\
\hline Tumor size $(>5 \mathrm{~cm} / \leq 5 \mathrm{~cm})$ & 2.103 & $0.847-5.220$ & 0.109 \\
Histological type (poor, muc/well, mod) & 1.429 & $0.566-3.604$ & 0.450 \\
Depth of invasion (T3, T4/T1, T2) & 1.330 & $0.544-3.250$ & 0.532 \\
Lymph node metastasis (present/absent) & 3.665 & $1.174-11.441$ & $0.025^{\text {a }}$ \\
Lymph node invasion (present/absent) & 1.371 & $0.602-3.121$ & 0.452 \\
Distant metastasis (present/absent) & 6.432 & $2.306-17.937$ & $<0.001^{\text {b }}$ \\
miR-20a (high/low) & 2.430 & $1.018-5.799$ & $0.045^{\text {a }}$
\end{tabular}

${ }^{\text {a }} \mathrm{P}<0.05$, ${ }^{\mathrm{P}} \mathrm{P}<0.01$. CI, confidence interval; well, well-differentiated; mod, moderately differentiated; poor, poorly differentiated; muc, mucinous; miR-20a, microRNA-20a.

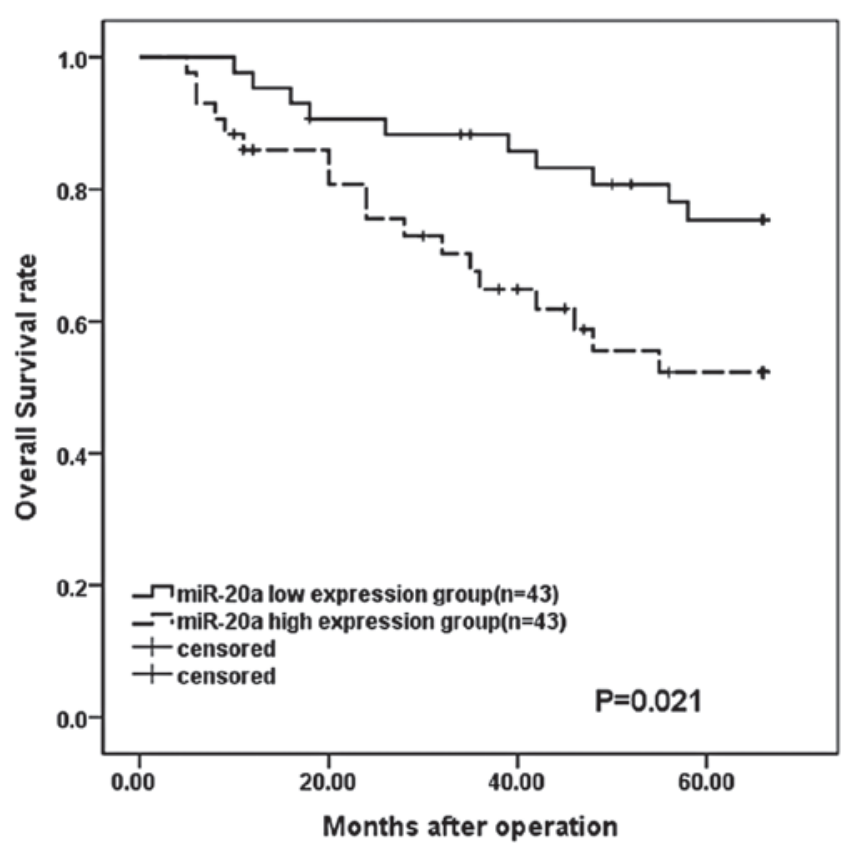

Figure 2. Kaplan-Meier survival curves of patients with colorectal cancer based on their miR-20a expression status. Patients in the high expression group had significantly more unfavorable prognosis than those in the low expression group ( $\mathrm{P}=0.021, \log$-rank test). miR-20a, microRNA-20a.

role of miR-20a in the migration and invasion of SW480 cells was examined (Fig. 3). Levels of miR-20a expression were observed to be significantly increased following transfection with pre-miR-20a compared with those following transfection with pre-miR-nc (Fig. 3A). Furthermore, cell migration was significantly increased following transfection with pre-miR-20a as compared with that following transfection with the negative control ( $\mathrm{P}<0.01$, Fig. 3B). The effect of miR-20a on SW480 cell invasion across an extracellular matrix was then assessed, revealing that the overexpression of miR-20a markedly enhanced cell invasion as compared with the control $(\mathrm{P}<0.01$, Fig. 3C). These observations suggested that miR-20a may have an important role in promoting migration and the invasive potential of CRC cells.

miR-20a induces EMT-like changes. pre-miR-20a-transfected SW480 cells were observed to undergo EMT-like transformation, as evidenced by the loss of cell-cell adhesion and alterations in morphology from a round, compact shape to an elongated, fibroblast-like morphology with scattered distribution, which may facilitate cell migration (Fig. 4A). In addition, western blot analysis indicated that overexpression of miR-20a downregulated the epithelial marker E-cadherin, while it upregulated the mesenchymal marker vimentin (Fig. 4B). Therefore, overexpression of miR-20a may induce EMT in SW480 cells.

SMAD4 is a target of $m i R-20 a$. Since miR-20a has a pivotal function in the metastasis of CRC cells, its putative target genes with metastatic functions were investigated using the online search tools TargetScan (http://www.targetscan.org) and miRanda (http://www.microrna.org). Among these genes, SMAD4, one of the mediators of transforming growth factor $\beta$ (TGF- $\beta$ ) signaling, was predicted to be a theoretical target of miR-20a based on putative target sequences at position 1,357-1,363 of the SMAD4 3'-UTR (Fig. 5A). Therefore, it was further investigated whether SMAD4 was the authentic target gene of miR-20a in CRC.

To directly address whether miR-20a bound to the 3'-UTR of the target mRNA, a luciferase reporter vector containing the SMAD4 3'-UTR with the putative miR-20a binding sites was generated. Correspondingly, a mutant reporter vector containing the SMAD4 3'-UTR with a mutation at the putative miR-20a binding site was produced. As shown in Fig. 5B, a marked reduction in luciferase activity was observed in cells transfected with pre-miR-20a as compared with pre-miR-nc-transfected cells $(\mathrm{P}<0.01)$. By contrast, no change in luciferase activity was observed in cells transfected with the mutant 3'-UTR constructs.

It was then determined whether the overexpression of miR-20a can lead to downregulation of SMAD4 protein expression. Western blot analysis showed that SMAD4 protein levels were markedly reduced in SW480 cells overexpressing miR-20a as compared with those in the control $(\mathrm{P}<0.01$, Fig. 5C). To further explore the correlation between miR-20a and SMAD4 expression in CRC tissue samples, miR-20a and SMAD4 mRNA were examined in 10 sets of CRC tissue samples. Using Pearson's correlation analysis, a significant inverse correlation between miR-20a and SMAD4 mRNA was observed $(P=0.033$, Fig. 5D). Taken together, the results 
B

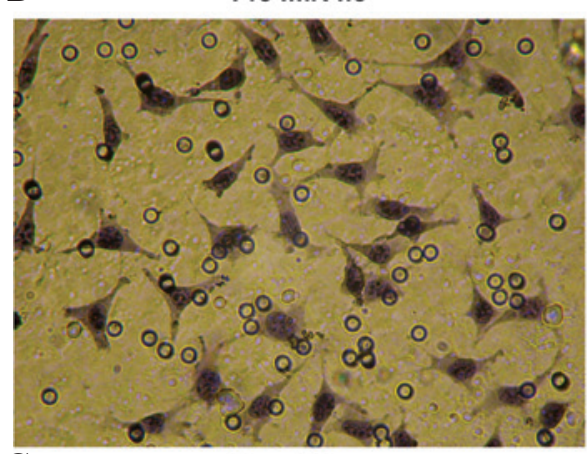

\section{C}

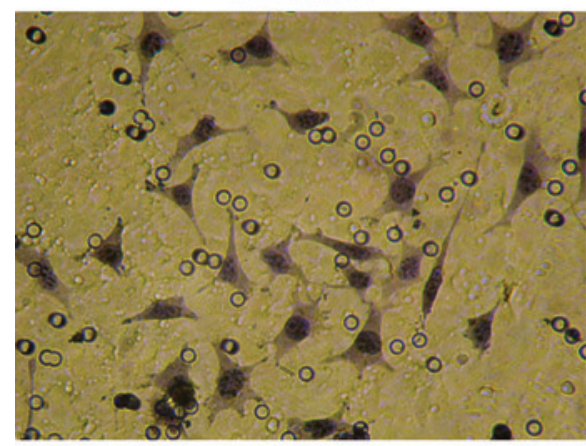

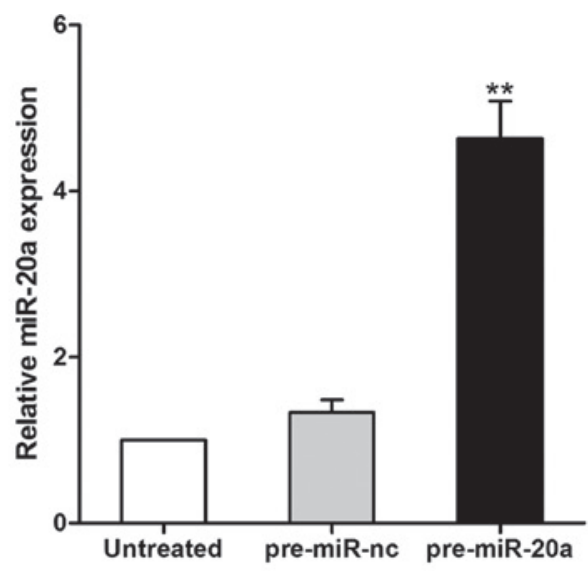
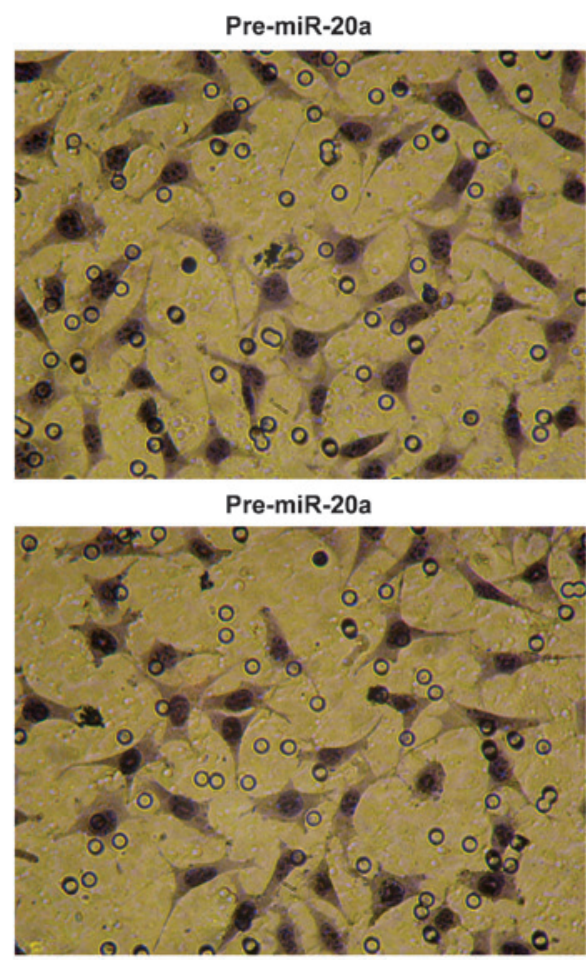
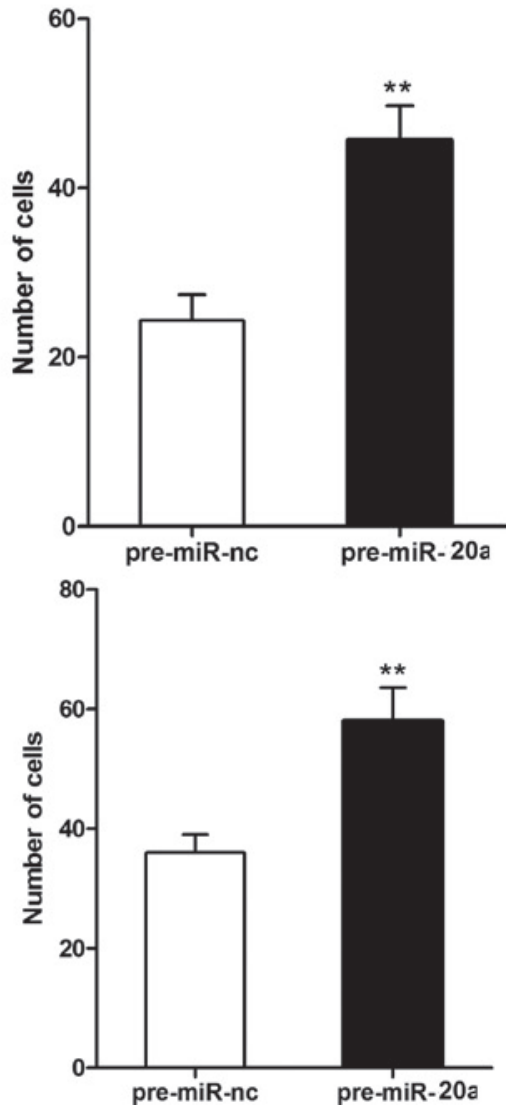

Figure 3. (A) miR-20a expression in SW480 cells transfected with pre-miR-20a or pre-miR-nc. miR-20a expression was determined by quantitative polymerase chain reaction. (B and C) Effects of miR-20a on the (B) migration and (C) invasive potential of SW480 cells (magnification, $x 400$ ). ${ }^{* *} \mathrm{P}<0.01$, pre-miR-20a versus pre-miR-nc. miR, microRNA; pre-miR-nc, negative control miRNA precursor; pre-miR-20a, miR-20a precursor.

A

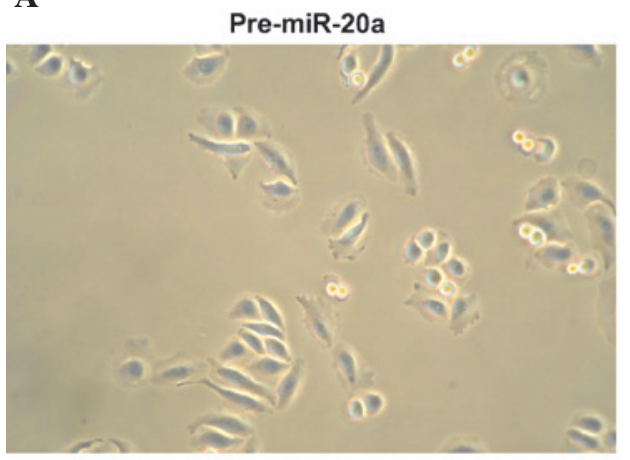

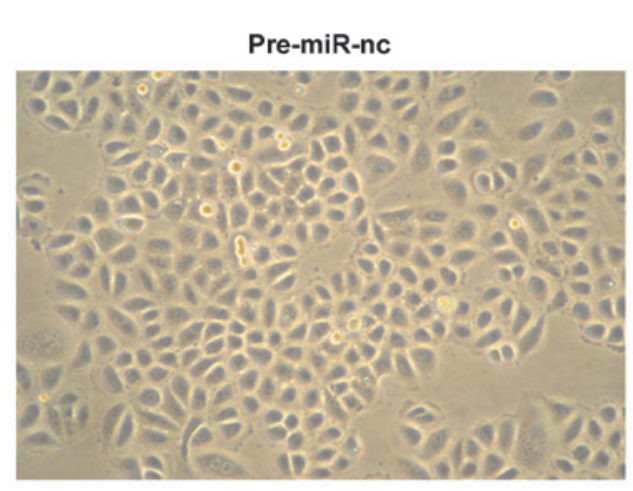

$\mathbf{B}$
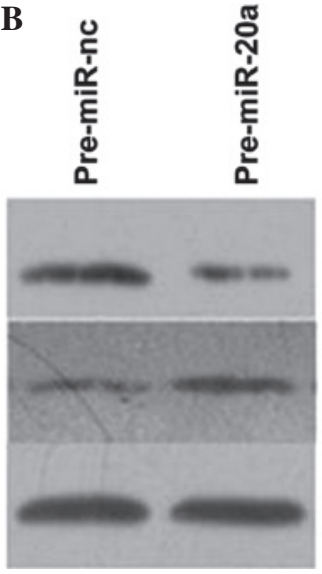

E-cadherin

vimentin

GAPDH

Figure 4. (A) Morphological changes in SW480 cells transfected with pre-miR-20a or the negative control (magnification, x400). (B) Expression levels of epithelial-mesenchymal transition markers (E-cadherin and vimentin) were measured by western blot analysis in pre-miR-20a-transfected and control SW480 cells. miR, microRNA; pre-miR-nc, negative control miRNA precursor; pre-miR-20a, miR-20a precursor. 
A

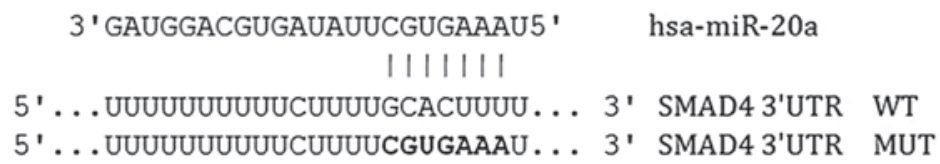

B

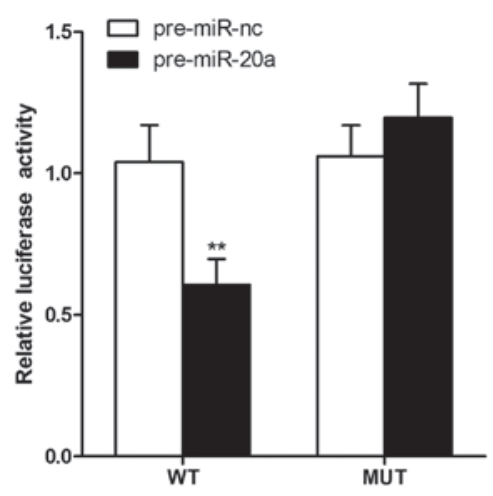

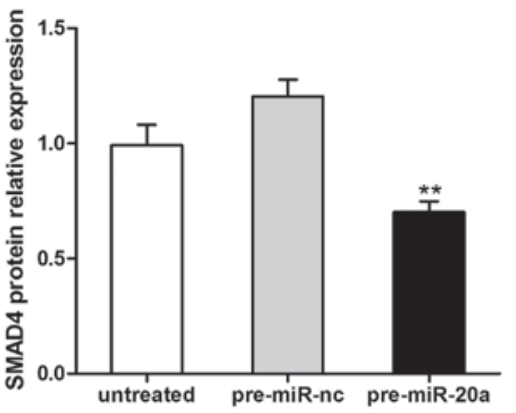

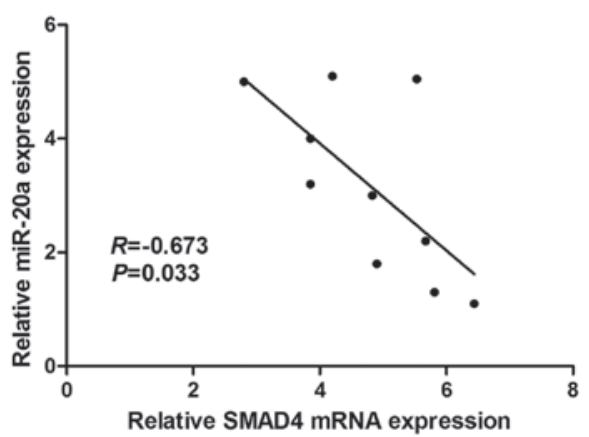

Figure 5. Identification of SMAD4 as a potential target of miR-20a in CRC. (A) WT and MUT 3'UTRs of SMAD4 with the seed region and base substitutions (bold). (B) HEK 293 cells were transiently co-transfected with luciferase reporter vectors and either pre-miR-20a or pre-miR-nc. Luciferase activities were normalized to the activity of Renilla luciferase. (C) Expression of SMAD4 protein was assessed using western blot analysis. (D) Correlation analysis between miR-20a and SMAD4 mRNA levels in CRC tissue samples (Pearson's correlation analysis). ${ }^{* *} \mathrm{P}<0.01$, pre-miR-20a versus pre-miR-nc. WT, Wild-type, MUT, mutated; miR, microRNA; UTR, untranslated region; SMAD4, SMAD family member 4; CRC, colorectal cancer; pre-miR-nc, negative control miRNA precursor; pre-miR-20a, miR-20a precursor.

suggest that miR-20a may attenuate the expression of SMAD4 by directly targeting the $3^{\prime}$ UTR of SMAD4.

\section{Discussion}

Accumulating evidence has indicated that dysregulation of miR-20a is associated with the development of cancer. However, the function of miR-20a in the development and progression of cancer is controversial. High levels of miR-20a have been found to correlate with malignant phenotypes and unfavorable prognosis in hepatocellular and gallbladder carcinoma $(19,20)$. Recently, it was shown that miR-20a encoded by the miR-17-92 cluster enhances the proliferation and metastatic potential of ovarian cancer and osteosarcoma cells $(21,22)$. By contrast, miR-20a overexpression inhibited proliferation and metastasis in breast and pancreatic cancers $(23,24)$. These controversial results may reflect the diverse roles of miR-20a in different types of cancer.

The present study demonstrated that miR-20a was frequently upregulated in CRC tissue samples as compared with normal adjacent mucosa, which was consistent with previous studies $(26,27)$. Furthermore, the patient group with high miR-20a expression levels showed a greater incidence of lymph node and distant metastases compared with the low miR-20a expression group, strongly suggesting that miR-20a may be involved in the metastasis of CRC. In addition, patients whose tumors had higher miR-20a expression levels had a significantly lower overall survival rate, indicating that high miR-20a levels are a marker of unfavorable prognosis for patients with CRC. Multivariate analysis indicated that high miR-20a expression was an independent prognostic factor for survival. However, Yu et al (26) reported that miR-20a was not an independent prognostic biomarker among the miR-17-92 cluster in colon cancer. This inconsistency may be due to differences in sample origin, tumor clinicopathological characteristics or different detection methods of the studies. Therefore, multi-institutional, prospective randomized trials are required before a consensus can be reached.

Given that miR-20a was upregulated in CRC tissue samples, it was speculated that miR-20a may have an oncogenic effect in CRC. As expected, ectopic expression of miR-20a promoted the migration and invasion of SW480 cells. In addition, the present study revealed that cells with high expression of miR-20a had a spindle-like morphology and that the upregulation of miR-20a decreased the expression of E-cadherin, while it increased the expression of vimentin, suggesting that miR-20a is involved in the regulation of EMT. The above data suggest that miR-20a is involved in modulating cell metastasis in CRC.

To address the molecular mechanisms involved in the miR-20a-mediated changes in biological properties, SMAD4 was selected for further study as it was predicted to be a target of miR-20a by bioinformatics analysis. SMAD4 is a member of the evolutionarily conserved family of SMAD proteins, which are transmitters of signals from the TGF- $\beta$ superfamily of cytokines (28). It has been suggested that SMAD4 can function as a tumor suppressor gene in gastrointestinal carcinoma $(29,30)$. The results in the present study indicated 
that SMAD4 is a direct target gene of miR-20a in CRC, since overexpression of miR-20a downregulated SMAD4 protein expression. Furthermore, miR-20a expression was inversely correlated with SMAD4 expression in CRC tumors, and overexpression of miR-20a significantly reduced the activity of a luciferase reporter containing the 3'UTR sequence of SMAD4. Combining these experimental results with the bioinformatics analysis led to the conclusion that SMAD4 is a target gene of miR-20a in CRC.

Loss of SMAD4 is considered to be a genetic step associated with the advanced stages of the disease, and frequently occurs in metastatic CRC (31). SMAD4 inactivation is rarely detected in adenomas; however, it strongly increases in frequency at late, metastatic stages $(32,33)$. Deckers et al (34) demonstrated that the knockdown of SMAD4 in breast cancer cells strongly attenuated the transformation of epithelial cuboidal cells into parallel aligned fibroblastic-like cells, as well as the downregulation of E-cadherin and the upregulation of N-cadherin, suggesting that SMAD4 acts as an EMT suppressor (34). Further studies have found a dependency of TGF- $\beta$-induced EMT on SMAD4 expression $(35,36)$. A previous study reported that the invasion suppressor E-cadherin is a target gene in the SMAD4 signaling network and re-expression of SMAD4 in SW480 human colon carcinoma induced E-cadherin expression (37). Pohl et al (38) reported that loss of SMAD4 promotes migration and invasion and mediates EMT in the SW480 CRC cell line. In the present study, restoration of miR-20a induced EMT and promoted CRC cell migration and invasion, which may be attributed to miR-20a-mediated downregulation of SMAD4 expression.

In conclusion, the present study has provided novel insights into the role of miR-20a in CRC. The results showed that miR-20a is an independent prognostic factor for patients with CRC and provided a potential mechanism for SMAD4 dysregulation and its contribution to CRC cell migration, invasion and EMT. miR-20a may function as a potential oncogene in CRC and has a potential application in cancer therapy.

\section{Acknowledgements}

The present study was supported by the Scientific Research Fund of Sichuan Provincial Education Department of China (CBY12-A-ZD16).

\section{References}

1. Tenesa A and Dunlop MG: New insights into the aetiology of colorectal cancer from genome-wide association studies. Nat Rev Genet 10: 353-358, 2009.

2. Yang IP, Tsai HL, Hou MF, et al: MicroRNA-93 inhibits tumor growth and early relapse of human colorectal cancer by affecting genes involved in the cell cycle. Carcinogenesis 33: 1522-1530, 2012.

3. Kalluri R and Weinberg RA: The basics of epithelial-mesenchymal transition. J Clin Invest 119: 1420-1428, 2009.

4. Thiery JP and Sleeman JP: Complex networks orchestrate epithelial-mesenchymal transitions. Nat Rev Mol Cell Biol 7: 131-142, 2006

5. Voulgari A and Pintzas A: Epithelial-mesenchymal transition in cancer metastasis: mechanisms, markers and strategies to overcome drug resistance in the clinic. Biochim Biophys Acta 1796: 75-90, 2009.

6. Du C, Zhang C, Hassan S, et al: Protein kinase D1 suppresses epithelial-to-mesenchymal transition through phosphorylation of snail. Cancer Res 70: 7810-7819, 2010.
7. Spaderna S, Schmalhofer O, Hlubek F, et al: A transient, EMT-linked loss of basement membranes indicates metastasis and poor survival in colorectal cancer. Gastroenterology 131: 830-840, 2006.

8. YaoD, Dai C and Peng S: Mechanism of the mesenchymal-epithelial transition and its relationship with metastatic tumor formation. Mol Cancer Res 9: 1608-1620, 2011.

9. Lee Y, Jeon K, Lee JT, et al: MicroRNA maturation: stepwise processing and subcellular localization. EMBO J 21: 4663-4670, 2002.

10. Lee Y, Ahn C, Han J, et al: The nuclear RNase III Drosha initiates microRNA processing. Nature 425: 415-419, 2003.

11. Lund E, Güttinger S, Calado A, et al: Nuclear export of microRNA precursors. Science 303: 95-98, 2004.

12. Esquela-Kerscher A and Slack FJ: Oncomirs - microRNAs with a role in cancer. Nat Rev Cancer 6: 259-269, 2006.

13. Li C, Nguyen HT, Zhuang Y, et al: Comparative profiling of miRNA expression of lung adenocarcinoma cells in two-dimensional and three-dimensional cultures. Gene 511: 143-150, 2012.

14. Moriyama T, Ohuchida K, Mizumoto K, et al: MicroRNA-21 modulates biological functions of pancreatic cancer cells including their proliferation, invasion, and chemoresistance. Mol Cancer Ther 8: 1067-1074, 2009.

15. Bhaumik D, Scott GK, Schokrpur S, et al: Expression of microRNA-146 suppresses NF-kappaB activity with reduction of metastatic potential in breast cancer cells. Oncogene 27: 5643-5647, 2008.

16. Zhang G, Xia S, Tian H, et al: Clinical significance of miR-22 expression in patients with colorectal cancer. Med Oncol 29: 3108-3812, 2012.

17. Paterson EL, Kazenwadel J, Bert AG, et al: Down-regulation of the miRNA-200 family at the invasive front of colorectal cancers with degraded basement membrane indicates EMT is involved in cancer progression. Neoplasia 15: 180-191, 2013.

18. Zhang GJ, Xiao HX, Tian HP, et al: Upregulation of microRNA-155 promotes the migration and invasion of colorectal cancer cells through the regulation of claudin-1 expression. Int J Mol Med 31: 1375-1380, 2013

19. Fan MQ, Huang $\mathrm{CB}, \mathrm{Gu} \mathrm{Y}$, et al: Decrease expression of microRNA-20a promotes cancer cell proliferation and predicts poor survival of hepatocellular carcinoma. J Exp Clin Cancer Res 32: 21, 2013.

20. Chang Y, Liu C, Yang J, et al: MiR-20a triggers metastasis of gallbladder carcinoma. J Hepatol 59: 518-527, 2013.

21. Fan X, Liu Y, Jiang J, et al: miR-20a promotes proliferation and invasion by targeting APP in human ovarian cancer cells. Acta Biochim Biophys Sin (Shanghai) 42: 318-324, 2010.

22. Huang G, Nishimoto K, Zhou Z, et al: miR-20a encoded by the miR-17-92 cluster increases the metastatic potential of osteosarcoma cells by regulating Fas expression. Cancer Res 72: 908-916, 2012.

23. Li JY, Zhang Y, Zhang WH, et al: Differential distribution of miR-20a and miR-20b may underly metastatic heterogeneity of breast cancers. Asian Pac J Cancer Prev 13: 1901-1906, 2012.

24. Yan H, Wu J, Liu W, et al: MicroRNA-20a overexpression inhibited proliferation and metastasis of pancreatic carcinoma cells. Hum Gene Ther 21: 1723-1734, 2010.

25. Chai H,Liu M, Tian R, et al: miR-20a targets BNIP2 and contributes chemotherapeutic resistance in colorectal adenocarcinoma SW480 and SW620 cell lines. Acta Biochim Biophys Sin (Shanghai) 43: 217-225, 2011.

26. Yu G, Tang JQ, Tian ML, et al: Prognostic values of the miR-17-92 cluster and its paralogs in colon cancer. J Surg Oncol 106: 232-237, 2012.

27. Motoyama K,Inoue H,Takatsuno Y,etal: Over-and under-expressed microRNAs in human colorectal cancer. Int J Oncol 34: 1069-1075, 2009.

28. Massagué $\mathrm{J}$ and Chen YG: Controlling TGF-beta signaling. Genes Dev 14: 627-644, 2000.

29. Wang LH, Kim SH, Lee JH, et al: Inactivation of SMAD4 tumor suppressor gene during gastric carcinoma progression. Clin Cancer Res 13: 102-110, 2007.

30. Powell SM, Harper JC, Hamilton SR, et al: Inactivation of Smad4 in gastric carcinomas. Cancer Res 57: 4221-4224, 1997.

31. Miyaki M, Iijima T, Konishi M, et al: Higher frequency of Smad4 gene mutation in human colorectal cancer with distant metastasis. Oncogene 18: 3098-3103, 1999.

32. Maitra A, Molberg K, Albores-Saavedra J and Lindberg G: Loss of Dpc4 expression in colonic adenocarcinomas correlates with the presence of metastatic disease. Am J Pathol 157: 1105-1111, 2000. 
33. Riggins GJ, Kinzler KW, Vogelstein B and Thiagalingam S: Frequency of Smad gene mutations in human cancers. Cancer Res 57: 2578-2580, 1997.

34. Deckers M, van Dinther M, Buijs J, et al: The tumor suppressor Smad4 is required for transforming growth factor beta-induced epithelial to mesenchymal transition and bone metastasis of breast cancer cells. Cancer Res 66: 2202-2209, 2006.

35. Valcourt U, Kowanetz M, Niimi H, et al: TGF-beta and the Smad signaling pathway support transcriptomic reprogramming during epithelial-mesenchymal cell transition. Mol Biol Cell 16: 1987-2002, 2005.
36. Levy L and Hill CS. Smad4 dependency defines two classes of transforming growth factor $\{$ beta\} (TGF-\{beta\}) target genes and distinguishes TGF-\{beta\}-induced epithelial-mesenchymal transition from its antiproliferative and migratory responses. Mol Cell Biol 25: 8108-8125, 2005.

37. Müller N, Reinacher-Schick A, Baldus S, et al: Smad4 induces the tumor suppressor E-cadherin and P-cadherin in colon carcinoma cells. Oncogene 21: 6049-6058, 2002.

38. Pohl M, Radacz Y, Pawlik N, et al: SMAD4 mediates mesenchymal-epithelial reversion in SW480 colon carcinoma cells. Anticancer Res 30: 2603-2613, 2010. 